\title{
Postmodernismus und Poststrukturalismus in der Geographie Einleitung zum Themenheft
}

\author{
Jürgen Hasse, Frankfurt am Main \\ Sabine Malecek, München
}

\begin{abstract}
«Räume» erscheinen als flüchtig, strittig und deshalb verständigungsbedürftig. Wichtige Instrumente zur Decodierung des Raumverstehens und -erzeugens leisten - neben der an Bedeutung gewinnenden phänomenologischen Erkenntnistheorie - die Gesellschaftstheorien, von denen jene aus dem theoretischen Felde des philosophischen Postmodernismus und des Poststrukturalismus in dem vorliegenden Heft im Vordergrund stehen. Die einzelnen Texte machen aus verschiedenen Perspektiven auf die Potentiale gesellschaftstheoretischer Ansätze zur Erklärung fundamentaler wie fallbezogener Beziehungen zwischen Menschen und ihren Umwelten aufmerksam.
\end{abstract}

Was Jean BAUdRILLARD kürzlich über die Architektur sagte, gilt generell für materiell manifestierte räumliche Strukturen: der ge- und bebaute Raum erzeugt - an den Grenzbereichen seiner Immaterialisierung - mehr als man sieht (vgl. BAUDRILLARD 1999: 11). Um verstanden, hand-habbar und be-greiflich zu werden, bedarf er der Interpretation. Diese ist situationsgebunden und in aller Regel auch sinngeleitet. Situationen, in denen sich ein Individuum befindet, sind zudem hinsichtlich Unentschiedenheit und Identität «chaotisch mannigfaltig» (vgl. Schmitz 1994: 68); die Richtungen der Orientierung sind mehr oder weniger offen. Schließlich erschwert sich die Interpretation als rein geistiger Akt dadurch, daß Individuen stets in zwei strukturverschiedenen wahrnehmungsspezifischen Einstellungen in eine Situation treten. Da ist zum einen das Individuum als leiblich (und implizit gefühlsmäßig) wahrnehmendes. Als solches ist es von einer spezifischen Befindlichkeit gleichsam eingerahmt. Man kann von einer ästhetischen Präsenz sprechen, die erkenntnistheoretisch weit über das hinausgeht, was zum Beispiel die Handlungstheorie als "körperliches» Dasein im physikalischen Sinne begreift (vgl. GidDENS 1984:65). $\mathrm{Da}$ ist zum anderen - im Normalfalle menschlicher Zurechnungsfähigkeit - das Individuum als gegenstands- und selbstbezüglich denkendes Individuum. Das Schisma zwischen Intellekt und Leib beziehungsweise Begreifen und Betroffensein (vgl. Schmitz 1998: 8 ) hat zivilisationsgeschichtliche Wurzeln, die mehr als zweitausend Jahre zurückgehen und heute im Sinne von Schmitz jene "Abstraktionsbasis" bilden, auf der wir uns in den Wissenschaften nach geschichtlichen Vorprägungen zu arrangieren gelernt haben. Schon die beiden elementaren erkenntnistheoretischen Eckpunkte der Leiblichkeit hier und der Intellektualität dort implizieren ja, daß die Dinge, Relationen, Situationen oder Konstellationen, die wir in der Geographie in den theoretischen Erklärungskontext von «Räumen» stellen, ihre Gültigkeit nur unter bestimmten Denkvoraussetzungen haben. Das Verhältnis Körper - Leib erweist sich nicht zuletzt auf dem Hintergrund der Beiträge dieses Heftes als Gegenstand der theoretischen Diskussion (vgl. dazu auch Hasse 1999).

\section{Philosophischer Postmodernismus}

Der vielbeschworene und -gescholtene philosophische Postmodernismus versteht sich in seinem seriösen, primär von Jean-Francois LyOTARD ausgehenden Werk als Menetekel der Vernunft (zu seiner Phänomenologie, aus der heraus sich die Kritik an der Moderne entfaltet, vgl. LYOTARD 1954, zur deutschsprachigen Diskussion in der Geographie vgl. HaSSE 1988, 1989. KRÜGER 1988 sowie kritisch dazu BeCKer 1996). Der philosophische Postmodernismus propagiert kein «anything goes»! Er reklamiert eine Erinnerung dialektischer Denkvermögen der Kritik. Die Grundwerte der Moderne sollen von dieser Kritik nicht mehr ausgeschlossen bleiben (vgl. LyOTARD 1982 sowie WELSCH 1987 u.a.). Herausragendes Merkmal ist ein (transversales) «Denken in Übergängen». Es trägt der strukturellen Vielheit weltkonstituierender Seinsweisen Rechnung, so daß diese nicht allein in synthetischer Sicht nebeneinander, sondern auch ineinander gesehen werden können. Besonders in den westlichen Metropolen laufen derzeit sozioökonomische Prozesse solcher Pluralisierungen durch Verinselung städtischer Räume ab. Das entstehende Patchwork wird dann nach Maßgabe gruppenspezifischer Identitätskonzepte als ein Gefüge gelebt, das räumlich durch Trajekte verbunden ist. Unübersichtlich ist uns auf dem Wege dieser Prozesse längst nicht mehr allein "das undurchschaubare System», sondern immer mehr unser Verhältnis zu uns selbst als die Hervorbringer gebrochener Weltsegmente.

Nicht zuletzt begegnet man jenen grenzüberschreitenden, sich eher durchdringenden als sich scharf gegeneinander abgrenzenden sozialen und räumlichen Formen in individuellen und gesellschaftlichen Beziehungen zur Natur. Natur können wir immer weniger von dem unterscheiden, was Menschen gemacht haben. Das betrifft auch das Verhältnis des «westlich» 
geprägten Menschen zu sich selbst als Natur. Der ist ja nicht nur physischer Körper im aristotelischen Sinne, sondern - auf einer phänomenologischen Ebene der Wahrnehmung - auch gefühlsmäßig spürendes Wesen im leiblichen und atmosphärischen Raum. Insbesondere die Moderne hat am Wissen der Menschen um das eigene nicht nur geistige Dasein in der Welt gezehrt. Dieser Prozeß des Vergessens hat die Wissenschaften nachhaltig geprägt. Die daraus entstandenen Sedimente des Denkens bilden heute eine zentrale Aporie des Postmodernismus. Für Wissenschaftlerinnen und Wissenschaftler heißt das (trotz aller Pluralität und Heterogenität), nicht nur über die Dinge nachzudenken, sondern forciert auch darüber, wie wir dies tun.

\section{Poststrukturalismus}

Poststrukturalismus fasziniert - in letzter Zeit auch immer mehr Geographinnen und Geographen. Und dies, obwohl er die wohlgeordnete Welt mit klaren Definitionen darüber, was Wissen und Wahrheit, was richtig und was falsch, was Natur und Kultur, was Subjekt und Identität sind, ganz gehörig durcheinanderwirft. Oder vielleicht gerade deshalb?

Ausgangspunkt für eine poststrukturalistische Herangehensweise ist der Konstruktivismus. Die Wirklichkeit und alle aus ihr resultierenden Kategorien werden als Ergebnisse sozialer Konstruktionen verstanden. Neben der Frage nach der Wissensproduktion werden auch Konstruktionsmechanismen der Gesellschaft in den Mittelpunkt des Interesses gerückt.

Der Poststrukturalismus war in seinen Anfängen eine Antwort auf den Strukturalismus. Dabei handelte es sich nicht nur um eine Antwort auf die unzureichenden Erklärungsmöglichkeiten des sozialen Wandels im Zusammenhang mit den Entwicklungen vor allem in Frankreich Ende der 60er Jahre, sondern «thinkers began also to react once more against the aspirations of modernism as a whole, hence, the postmodern aspect to poststructural philosophy» (PEET 1998: 195). Poststrukturalistisches Denken nimmt seinen Ursprung in der Ablehnung der Stabilität und Fixiertheit aller sozialen Beziehungen und stellt jegliches fixe Konzept sowie die Sicherheit allen Wissens in Frage. Der Poststrukturalismus versucht, das Verhältnis zwischen Subjektivität, Bedeutung und gesellschaftlichem Wert zu erklären.

Zentrales Element aller poststrukturalistischer Ansätze ist die Sprache. «Sie wird als der Ort verstanden, wo tatsächliche und mögliche Formen der gesellschaftlichen Organisation und ihre sozialen und politischen Konsequenzen definiert und in Frage gestellt werden. Sie ist aber auch der Ort, an dem unsere Eigenwahrneh- mung, unsere Subjektivität konstruiert ist und schließt mit ein, daß sie nicht angeboren, nicht genetisch determiniert, sondern ein Produkt der Gesellschaft ist» (WeEdon 1991:35). Die Sprache ist somit kein Spiegel gesellschaftlicher und natürlicher Realitäten, sondern diese werden erst über Sprache hergestellt.

Im Zentrum wissenschaftlicher Analysen stehen weniger Tatsachen als solche, sondern jene Mechanismen, die eine Tatsache zu einer ebensolchen machen. Zentrales Element ist hier die Diskurstheorie Michel FouCAULTs (vgl. 1978 oder 1987). Mit einem Diskursbegriff, der nicht auf sprachliche Aussagesysteme reduziert ist, sondern um den im jeweiligen zeitlichen und räumlichen Kontext geltenden gesellschaftlichen Ordnungsrahmen erweitert ist, wird es möglich, soziale Phänomene als Produkt gesellschaftlicher Strukturen zu erklären. Dabei wird ein Zusammenhang zwischen Macht, einer spezifischen Diskursformation und der Produktion von Wahrheit hergestellt (Foucault 1978: 76). Daraus ergibt sich die Möglichkeit, die gesellschaftliche Realität als diskursiv produziert zu entlarven und eine Vorstellung von anderen Wirklichkeiten jenseits der hegemonialen, scheinbar unveränderbaren Ordnung zu erhalten. Nach Jacques Derrida (1976) beruhen die Denksysteme westlicher Kultur auf binär hierarchisch geordneten Gegensatzpaaren. Die Dekonstruktion ermöglicht es, diese durch Dezentralisieren und Pluralisieren aufzulösen.

Soziale Konstruktionen spielen auch in den feministischen Diskussionen eine zentrale Rolle. Neben der Thematisierung der Konstruktion der Geschlechter werden in feministisch-geographischen Ansätzen auch gesellschaftlich manifeste, jedoch konstruierte Raumstrukturen und die ihnen zugrunde liegenden Machtverhältnisse thematisiert. Gemeinsam mit der Denaturalisierung der Geschlechterdifferenz wird auch der Körper als diskursiv produziert verstanden (BUTLER 1991,1997). In den Analysen werden nicht nur die Auswirkungen von Strukturen auf einzelne Mitglieder der Gesellschaft untersucht, sondern es geht in der poststrukturalistischen Variante vielmehr um das Aufdecken der diversen Diskurse, die die bestehenden Gesellschafts- und Geschlechterverhältnisse sowie Raumstrukturen produzieren, reproduzieren und dadurch stabilisieren.

Und gerade hier spielt der Aspekt der Körperlichkeit eine ganz wesentliche Rolle. Es hat Konsequenzen für unser Verständnis von Macht, Wissen sowie der Beziehungen zwischen Mensch und Raum, wenn wir den sexualisierten Körper als kritische Komponente in die wissenschaftliche Theoriebildung mit einbeziehen. Es geht dabei darum aufzuzeigen, auf welche Weise sexualisierte Körper sich im patriarchal strukturierten Raum bewegen (Rose 1995: 546) und damit verbun- 
den auch um die Frage, wer in welcher Form zu welcher Zeit richtig oder fehl am Platz ist.

Da diskursive Praktiken als prozeßhaft verstanden werden, beinhaltet die ständige Neukonstruktion auch Möglichkeiten zur Veränderung scheinbar dauerhaft bestehender Strukturen. Gesellschaftliche Aushandlungsprozesse um Räume und die Aneignung von Räumen geben unterschiedlichen Personen beziehungsweise Gruppen - und hier vor allem jenen, die im bestehenden hegemonialen System benachteiligt werden oder auch nicht sichtbar sind oder sein sollen - die Möglichkeit, Raum zu greifen und an der Gestaltung räumlicher Strukturen aktiv teilzuhaben.

\section{Bedeutung für die Geographie?}

Die theoretischen Denkansätze zeigen eine Vielfalt von Möglichkeiten die Welt anders zu denken. Daraus ergibt sich ein Nachdenken über Texte und Diskurse und darüber hinaus über SprecherInnenpositionen, Wahrnehmung, Beobachtung und Darstellung unterschiedlicher Wirklichkeiten. Dies hat Konsequenzen für die Gestaltung unserer Gesellschaftsverhältnisse, aber auch für die Produktion von Wissen und von Wahrheit.

Darin liegt auch eine Chance für die Geographie. Ihre großen Themen «Raum», «Landschaft» oder «Natur» können unter veränderten Prämissen gedacht werden. Die Denkansätze, die das Relationale sowie die Vernetzung in den Mittelpunkt stellen, eröffnen neue Möglichkeiten zur Konzeptionalisierung von Natur oder von Landschaft bzw. des Verhältnisses von Natur zu Kultur oder von Gesellschaft zu Raum.

Wege eines in diesem Sinne differenzfreudigen Denkens, das über tradierte Alternativen hinausgeht, weisen die Beiträge dieses Heftes. Aus verschiedenen Perspektiven machen sie den Standort des Menschen in Welt- und Erkenntnisprozessen zu ihrem Thema. Dabei geht es um Wahrnehmungs- und Aneignungsweisen von Räumen. Jede Einlassung auf die Wahrnehmung und das praktische Tun von Menschen überschreitet notwendig die Enge kognitivistischer Menschenbilder. Der idealistische Entwurf des handelnden Menschen, wie er etwa durch GidDENS (1984) begründet und durch WerLeN (1987) auf die Geographie übertragen wurde, rückt aber nicht nur deshalb in den Argumentationskontext postmodernistischer und poststrukturalistischer Gesellschaftstheorien, sondern auch wegen der Annektion des Begriffes «Kritischer Theorie» durch GIDDENS - stand «Kritische Theorie» doch als Synonym für eine Vielzahl noch heute aktueller Studien der Frankfurter Schule (insbesondere von Horkheimer, Adorno, Marcuse und dem frühen Habermas). Mit dem in geisteswissenschaftlicher Perspektive in vielfacher Hinsicht strittigen Ort des Einzelnen steht das Selbst-Bewußtsein des (post)modernen Subjekts/Objekts/Individuums im Mittelpunkt der Frage nach der fachtheoretischen Erklärung der Beziehungsstrukturen, die sich zwischen Menschen und ihren Umwelten aufspannen. Inwieweit der Mensch sich neben seinem Handeln nicht durchaus auch - der Gründe seines Tuns nicht sehr bewußt verhält, ist einstweilen offen (zur Kritik an der Handlungstheorie vgl. zum Beispiel MeusBurger 1999).

Wissenschaft kann sicherlich nie unter zu großer Intellektualität leiden, sehr wohl aber an einem zu großen Affekt gegen das Gefühl, obgleich es doch eine evaluative Rolle im Leben spielt und das (kognitiv) Denkbare imprägniert. Menschen agieren nicht nur in einem (singulären) Sinne rational, sondern in einem pluralen. Deshalb sprechen die Geisteswissenschaften neben theoretischer auch von ästhetischer Rationalität und die Psychologie von Affektlogik. In der einen wie der anderen Perspektive agiert der Mensch nicht als kognitivistisches Hyperbrain, sondern stets aus situativen Ganzheiten, in denen gerade das leibliche Befinden und gefühlsmäßige Erleben einen bewertenden und richtungsgebenden Einfluß auf intelligentes Denken nimmt.

Die in der Geographie heute verbreiteten kognitionslastigen Menschenbilder mögen wissenschaftstheoretisch und abstraktionshygienisch taugliche Konstrukte sein; sie können den Differenzierungen aktueller gesellschaftlicher und sich in räumlichen Substraten widerspiegelnden Entwicklungen (auch den darin zur Geltung kommenden Entfremdungspotentialen) kaum gerecht werden. Die Idealisierung eines in seinem Tun voll und ganz rechtfertigungsfähigen Menschen hinterläßt blinde Flecken in der Analyse sozialräumlicher Prozesse. Mit einer szientistischen Umschiffung diffuser Beziehungsstrukturen im Mensch-Umwelt-Verhältnis ist niemandem gedient. Solange die Humangeographie die Erklärung so diffiziler Sachverhalte wie zum Beispiel Heimat, Landschaft oder Mobilität anstrebt, wird sie an einer kritischen Reflexion und Revision methodologisch einund vorausgesetzter Menschenbilder nicht vorbeikommen.

\section{$4 \mathrm{Zu}$ den einzelnen Beiträgen}

Eine wissenschaftstheoretische Einordnung, Dezentrierung und Pointierung postmodernen Denkens leistet Wolfgang Zierhofer mit dem ersten Beitrag. Im Rückgriff auf Systemtheorie und Sprachpragmatik wird die erkenntnistheoretisch ein- und ausgrenzende Funktion begrifflicher Abstraktionen (oft in der 
Gestalt wissenschaftshistorisch tradierter Schismen) aus der Perspektive relationalen Denkens aufgehellt. Dieses «nachmetaphysische» Denken operiert ohne vorausgesetzte theoretische Fixpunkte. Analyse- und Bezugspunkt der Abhandlung ist die (Schein-) Dichotomie von Kultur und Natur. ZIERHOFER zeigt, daß die systemtheoretische Funktion der wissenschaftlichen Disziplinen stets darin bestand, Beobachtungsfähigkeit und Selektivität weiterzuentwickeln. Die in kritischer Sicht überfällige Auflösung der tradierten Dichotomie von Kultur und Natur vermittelt der Geographie unter der Voraussetzung eine konstruktive Forschungsperspektive, daß sie sich auf der Basis a-modernen Denkens an den Grenzverläufen «hybrider Forschungsgegenstände» orientiert, um so ihr theoretisches Erklärungs- und praktisches Problemlösungspotential zur Seite der Natur- und der Sozialwissenschaft hin öffnen zu können.

Der Vermengung von Kultur und Natur widmen sich auch die folgenden drei Beiträge. An illustrierten Beispielen thematisiert Jürgen STRassel Gärten als räumliche Texte, die als widerspruchsglättende Mythen decodiert werden. Der Verfasser geht von der These aus, daß gegenwärtig erfahrbare Brüche im Natur-Kultur-Verhältnis in einer pluralen Ästhetik des Gartens ausgeglichen werden. Der Garten (im allgemeinen) wird so als «zeitgemäße Antwort auf eine menschliche Sehnsucht von heute» begreiflich. Der Beitrag legt diskursive Praxen offen, welche sich meta- oder sondersprachlich artikulieren und in ihren jeweiligen Formen Wege der sozialen Manövrierung in einer von tiefen Antagonismen geprägten Gesellschaft offerieren. Strassel zeigt, daß Gärten erst weit jenseits dessen zur Sprache kommen, was man materiell «als solche» sieht. STRASSEls diskursanalytische Entzifferung von Gärten weist auch auf ein Verschwinden ästhetischer Wirklichkeiten unter der Vorherrschaft des theoretischen Erkennens hin. In diesem Grabenbruch generiert sich die Sprache der Gartengestaltung als eine interdiskursive Vermittlungsform individuellen Wohlergehens und herrschender Formen des gesellschaftlichen Umgangs mit Natur.

Die beiden folgenden Beiträge legen die Auswirkungen herrschender Geschlechterkonstruktionen in räumlichen Kontexten offen. Dabei zeigt sich die Praxisrelevanz poststrukturalistischer Ansätze, da auf eine Dekonstruktion immer eine Rekonstruktion folgt. Dadurch bleibt die symbolische Belegung von Räumen nicht per se festgeschrieben, sondern es besteht eine aktive Möglichkeit zur Raumaneignung gerade für jene Individuen und Gruppen, die im herrschenden Diskurs als zu bestimmten Zeiten an bestimmten Orten auch aufgrund ihrer körperlichen Merkmale (Hautfarbe, Geschlecht, Alter...) fehl am Platz angesehen werden. So sind Räume einer ständigen Neukon- struktion unterworfen, die auch in gesellschaftliche Aushandlungsprozesse um Macht in und über Räume mündet.

In dem Gemeinschaftsbeitrag von Sybille BauriedL, Claudia WuChERPFEnNig, Anke STrüver und Katharina FleischmanN wird der Zusammenhang von Körpern und Räumen aufgezeigt. Auf theoretischer Ebene wird das Raumkonzept von Pierre Bourdieu (1991) nachgezeichnet und mit den Überlegungen Judith BuTLERs (1991,1997) und Elisabeth Lists (1994) verknüpft. Um die Mechanismen der Konstruktionen von Körpern in Räumen besser verständlich machen zu können, erfolgt eine analytische Trennung der Wahrnehmung von Körpern in Eigen- und Fremdwahrnehmung. Anhand des für die Geographie ungewöhnliche Beispiels von an Brustkrebs erkrankten Frauen, wird das Wechselspiel zwischen Fremd- und Eigenkonstruktion von Körpern im Raum gut sichtbar. Es werden Ansatzpunkte dafür gegeben, wie es durch das durch die Dekonstruktion gewonnene Wissen über Konstruktionsmechanismen ermöglicht wird, diese aufzubrechen und anders zu gestalten.

Eine unkonventionelle Analyse des Themas «Angsträume» bietet der Beitrag von Karin KUTSCHINSKE und Verena Meier. Über Angsträume wird seit den Anfängen feministisch geographischer Analysen viel nachgedacht und geschrieben. Der vorliegende Beitrag nähert sich der Thematik von einer völlig anderen Seite als der Großteil der Arbeiten hierzu. Die Autorinnen verstehen die Produktion von Angsträumen als einen Ausdruck der Geschlechterkonstruktionen in den herrschenden Gesellschaftsverhältnissen. Sehr schön werden die einzelnen Diskursstränge über Angst, Sicherheit und Rollenverständnis aufgezeigt. Es wird analysiert, wie Frauen/angst/raumkonstruktionen funktionieren und welche Diskurse an der Festschreibung derselben beteiligt sind. Dabei wird deutlich, daß die vorherrschenden wissenschaftlichen Diskurse über Frauen/angst/räume sich weniger der Ursache, als der Wirkung des Problems annehmen. In diesem Beitrag wurde ein Beispiel gewählt, das Frauen nicht zu Opfern, sondern zu aktiv Handelnden macht. Anhand von Frauen, die bei Dunkelheit an der Isar in München joggen, wird gezeigt, wie durch das Laufen eine Rekonstruktion einzelner Diskurse gemacht wird und wie aus dem ursprünglich dunklen, einsamen nächtlichen Isarufer ein angeeigneter Freiraum entstehen kann.

\section{Literatur}

BAUDRILLARD, J.(1999): Architektur:Wahrheit oder Radikalität? - Essay 40, Graz: Literaturverlag Droschl.

Becker, J. (1996): Geographie in der Postmoderne? Zur Kritik postmodernen Denkens in Stadtforschung 
und Geographie. $-=$ Potsdamer Geographische Forschungen 12, Potsdam.

Bourdieu, P. (1991): Phyischer, sozialer und angeeigneter physischer Raum. - In: WENTZ, M. (Hrsg.): StadtRäume. - Frankfurt am Main: 25-34.

Burler, J. (1991): Das Unbehagen der Geschlechter. Frankfurt am Main: Suhrkamp.

BUtLER, J. (1997): Körper von Gewicht. - Frankfurt am Main: Suhrkamp.

Derrida, J. (1976): Die Schrift und die Differenz. Frankfurt am Main: Suhrkamp.

Foucault, M. (1978): Dispositive der Macht: über Sexualität, Wissen und Wahrheit. - Berlin: Suhrkamp.

FoucAult, M. (1987): Das Subjekt und die Macht. - In: DreYfus, H. et al.: Michel Foucault. Jenseits von Strukturalismus und Hermeneutik. - Frankfurt am Main: Athenäum Verlag.

Giddens, A. (1984): The Constitution of Society. Oxford: Polity Press.

HASSE, J. (1988): Die räumliche Vergesellschaftung des Menschen in der Postmoderne. - = Karlsruher Manuskripte zur Mathematischen und Theoretischen Wirtschafts- und Sozialgeographie 91, Karlsruhe.

HASSE, J. (1989): Sozialgeographie an der Schwelle zur Postmoderne. Für eine ganzheitliche Sicht jenseits wissenschaftstheoretischer Fixierungen. - In: Zeitschrift für Wirtschaftsgeographie 1/2,33. Jg.: 20 - 29.

Hasse, J. (Hrsg.) (1999): Zum Verhältnis von Raum und Gefühl in der Anthropogeographie. - Themenheft der Geographischen Zeitschrift 2, 87. Jg.

Krüger, R. (1988): Die Geographie auf der Reise in die Postmoderne? - = Wahrnehmungsgeographische Studien zur Regionalentwicklung 5, Oldenburg: BIS Verlag.

List, E. (1994): Wissende Körper - Wissenskörper Maschinenkörper. - In: Die Philosophin 5: 9-26.

Lyotard, J.-F. (1954): Die Phänomenologie. - Hamburg 1993: Junius.

LYOTARD, J.-F. (1982): Das postmoderne Wissen. - Wien 1986: Böhlau.

Meusburger, P. (1999): Subjekt-Organisation-Region. Fragen an die subjektzentrierte Handlungstheorie. In: Meusburger, P. (Hrsg.): Handlungszentrierte Sozialgeographie. Benno Werlens Entwurf in kritischer Diskussion. - In: Erdkundliches Wissen 130: 95-132.

PeET, R. (1998): Modern geographical thought. Oxford: Blackwell.

Rose, J. (1995): Geography and Gender, Cartographies and Corporealities. - In: Progress in Human Geography 4: 544-548.

Schmiтz, H. (1994): Neue Grundlagen der Erkenntnistheorie. - Bonn: Bouvier.

Schmitz, H. (1998): Der Leib, der Raum und die Gefühle. - Ostfildern: Tertium.

WeEdon, C. (1991): Wissen und Erfahrung. Feministische Praxis und poststrukturalistische Theorie. Zürich: efef.
WeLSCH, W. (1987): Unsere postmoderne Moderne. Weinheim: Acta Humaniora.

WerLen, B. (1987): Gesellschaft, Handlung und Raum. Grundlagen einer handlungstheoretischen Sozialgeographie. - Stuttgart: Franz Steiner Verlag.

\section{Zusammenfassung: \\ Postmodernismus und Poststrukturalismus in der Geo- graphie - Einleitung zum Themenheft}

Raum und räumliche Beziehungen sind Konstrukte. Sicht-, Denk- und Interpretationsweisen wirken aus dem Hintergrund der aktuellen und historisch gewachsenen gesellschaftlichen Verhältnisse auf die Herstellung nicht zuletzt sprachlich konstruierter Wirklichkeiten ein. In diesem Beitrag werden die Grundzüge von Poststrukturalismus und Postmodernismus skizziert und das vorliegende Heft in seinem Stellenwert für die (Human-) Geographie begründet. Desweiteren wird in die einzelnen Beiträge des Heftes eingeführt.

\section{Summary: Postmodernism and Poststructuralism in Geography - Introduction to this special issue}

Space and spatial relationships are social constructions. The way we see, think and interpret worldly activity and phenomena influences the construction of our personal reality, foremost a linguistic reality, and is embedded in current and historical social relations. The basics of postmodernism and poststructuralism are outlined in this paper, which serves as an introduction to the articles following. At the same time, their relevance is highlighted.

Résumé: Postmodernisme et poststructuralisme en géographie - introduction à la revue thématique

L'espace et les relations spatiales sont des constructions. Les façons de voir, de penser et d'interpréter, qui sont influencées par les conditions sociales actuelles et historiques, agissent sur la production des réalités construites linguistiquement. Cet article esquisse les principaux traits du poststructuralisme et du postmodernisme et présente les différents articles de la revue, tout en expliquant leur importance pour la géographie humaine.

Prof. Dr. Jürgen Hasse, Fachbereich Geographie, Johann Wolfgang Goethe-Universität Frankfurt a/Main, Schumannstrasse 58, D-60054 Frankfurt am Main. e-mail: J.Hasse@em.uni-frankfurt.de

Sabine Malecek M.A., Geographisches Institut, TU München, Arcisstrasse 21, D-80290 München.

e-mail: sabine.malecek@ws.tum.de 